\title{
A Framework to Enhance Performance of E-Shopping
}

\author{
N. Jayakanthan, M. Manikantan, R. Rassika
}

\begin{abstract}
E-shopping is a trend in present scenario. Today the shopping in internet is become a culture and habit of people. Lot of E-commerce merchants are available in market. But the online shopping system suffers a lot with various issues like performance overhead, slow response, late and error prone deliveries. Hence it is essential to enhance the performance on the online shopping system. In this paper we propose a model "Shop IT" to address the above issues. It uses greedy based "Route Mapper Algorithm" to find the shortest route between the cities and constraint based "Optimum Grouping" algorithm to group the items in the appropriate cluster. The proposed algorithm solves the performance issues.
\end{abstract}

Keywords - Online shopping, Clustering, Shortest path, Optimum grouping

\section{INTRODUCTION}

The e-ecommerce is a useful tool for general public and industries. It is become the part of the human life. Various research activities are carried out in this area. The aspects of the research address various areas like improve the supply chain, stream line the selling chain, optimize the delivery time, and reduce the order errors. But still e-commerce industry not able to fulfill the expectation level of the customer. This opportunity motivates various researchers and work in this area.

The existing quality models are not able to address the emerging issues of the e-commerce. Lot of vulnerabilities is available in the system. Grouping items as cluster and transporting them towards shortest route is a challenging issue.

This paper proposes "Shop IT". "A Route Mapper" algorithm has developed to find the shortest route. Optimum grouping algorithm is used to group the items in the appropriate clusters. The proposed algorithms address the grouping and routing issues.

The rest of the chapter organizes as follows chapter 2 provide Survey of Literature, chapter 3 represent the algorithms and analysis of the result is given in the chapter 4. Chapter 5 conclude the research work.

\section{SURVEY OF LITERATURE}

Abdul Gaffar Khan [1] analyzes benefits and challenges in E-commerce economy. It analyzes various parameters of customer satisfaction. The advancement of information and

Revised Manuscript Received on August 14, 2019.

N. Jayakanthan, Assistant Professor(SRG), Department of Computer Applications, Kumaraguru College of Technology Coimbatore T.N, India. (E-mail: jayakanthan.n.mca@kct.ac.in)

M. Manikantan, Assistant Professor(SRG), Department of Computer Applications, Kumaraguru College of Technology Coimbatore, T.N, India. (E-mail: manikantan.m.mca@kct.ac.in)

R. Rassika, PG Scholar, Department of Computer Applications, Kumaraguru College of Technology, Coimbatore. T.N, India. (E-mail: rassika.16mca@kct.ac.in) communication technologies brings lot of challenges. The study analyzes lot of challenges in emerging economy.

Bhupinder Singh et al [2] analyze the impact of technology in e-commerce. The e-commerce security mechanism. The significance of the computing time in ecommerce.

Nisha Chanana et al [3] discuss the future of E-commerce in Indian. This paper articulate the overall growth of the ecommerce will increase in forth coming years.

D. K. Gangeshwer [4] discusses various motivational factors of shopping online. The current development is a valuable increase in the present scenario.

M.Shirisha et al [5] analyze the impact of the digital marketing in India. This paper analyzes the impact of the digital marketing campaign in real-time.

Diyan Ivanov [6] analyse the various limitation in ecommerce implementation in current scenario. He addresses various drawbacks like security, lack of qualified people and interoperable systems in e-commerce.

Lokesh Giripunje [7] analyzes the security issues in mobile devices which impacts the mobile commerce or Mcommerce.

N. Jayakanthan et al [8] proposed a model to improve the performance and latency issues in mobile commerce. Clustering and scheduling algorithms are used for grouping.

N. Jayakanthan et al [9] proposed a model which analyses various security issues in e-commerce and websites. The suggestions are helpful to further improvement.

\section{METHODOLOGY}

To improve the performance of the e-commerce application are the essential task. In this research work an effective system "Shop IT" is proposed. It considers the location and items are the input, and create cluster. The source and destination cities are used to this tool contains two parts. In first part a greedy method based algorithm. "A Route Mapper" is proposed. It finds the shortest path between various cities. The proposed algorithm computes the clustering and shortest distance.

In the State-I A Route Mapper Algorithm is used. It is a greedy method based algorithm. It finds the shortest route through various cities for delivery.

\section{1) Stage-I Route Mapper Algorithm}

Input: A (X, Y) Each Vertex with nearest Vertex and its Weight.

Output: Optimum path between the cities.

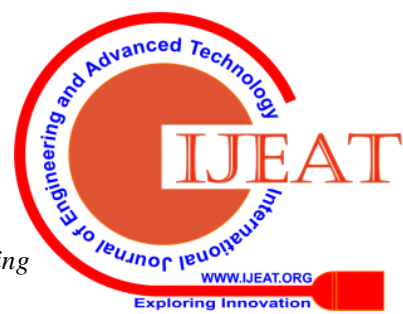


1. Randomly chose a vertex as initial vertex.

2. Add it in the binary tree $(\mathrm{S})$.

3. Compute the remaining distance and priorities of all vertexqueue (S_SX) which are nearest to the tree vertex.

4. Order the Vertices based on their weight.

5. Chose the minimum Vertex in the queue and add it in the tree.

6. Repeat the steps the graph until is empty.

In stage - II Optimum Grouping algorithm is used for clustering.

\section{2) Stage-II Optimum Grouping Algorithm}

Input: Item No \& City.

Output: Optimal Clusters.

1. Get the item IT \& City- $\mathrm{C}_{\mathrm{T}}$.

2. Create a cluster $\mathrm{C}_{\mathrm{i}}$ based on the city.

3. Pick the item and add in the cluster.

4. Repeat the process all the items IT belongs to the concerned city $\mathrm{C}_{\mathrm{T}}$ is empty

5. Create the next cluster $\mathrm{C}_{\mathrm{i}}$ and add the items.

6. Repeat the entire process until all items of empty.

7. Call shortest path algorithm to fix the mapping.

In the stage -II optimum grouping algorithm is used to create clusters. It takes the city and item belongs to the city as input. It creates an initial cluster and arrange all items belongs to the clusters. If the items belong to the clusters are empty, next the next cluster is created. The process got repeated until all items are empty. After the formation of the clusters the Route Mapper Algorithm is used to form the shortest path.

The clustering and Route Mapper Algorithm is used. The clustering algorithm efficiently used to form the groups. After the formation of the clusters and the shortest route between the cities are used for transportation. The proposed system addresses the drawback of the present scenario.

\section{IMPLEMENTATION AND EVALUATION RESULTS}

The "Shop IT" tool is implemented in Java. In the Stage I Route Mapper Algorithm is used to find the shortest path between cities and the edge weights are used to calculate the path length. For clustering optimum grouping algorithm is used. It takes items and concerned cities to form the clusters. Once the clusters are formed the shortest path between cites are framed. Finally, a route map is generated for delivery. The algorithm is implemented as separate classes of the Java project. Few real time sources are used in data collection. Few data are formed as a synthesized data. Disconnect data set is used for to train and test the system to improve the efficiency.

The algorithm generates various clusters. Few provinces in United States are analyzed. The groups are given in the figure 1. Dallas reported $39 \%$ of the clusters. $19 \%$ of the clusters are generated for Washington. 13\% for Seattle. Boston reports $2 \% .17 \%$ of the clusters are generated for SanDiego.

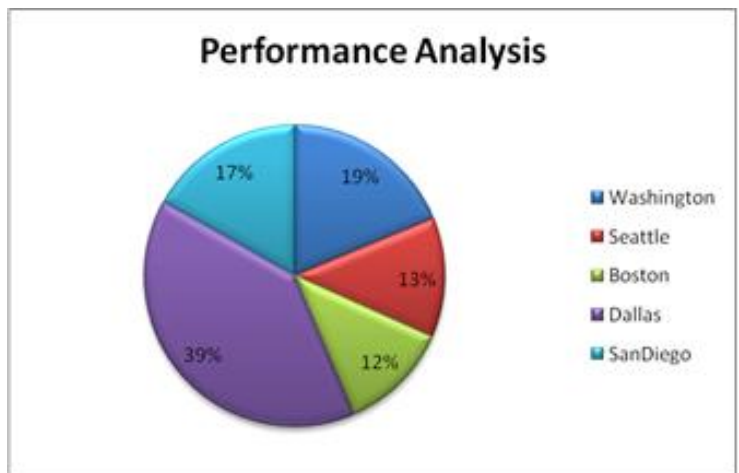

Figure 1 Provinces and Clusters in USA

Our tool "Shop IT" is compared with K-Mean clustering and support vector machine. Data sets of size 600 are analyzed. The experimental results are shown in the figure2. The proposed algorithm generates clusters efficiently than other two algorithms.

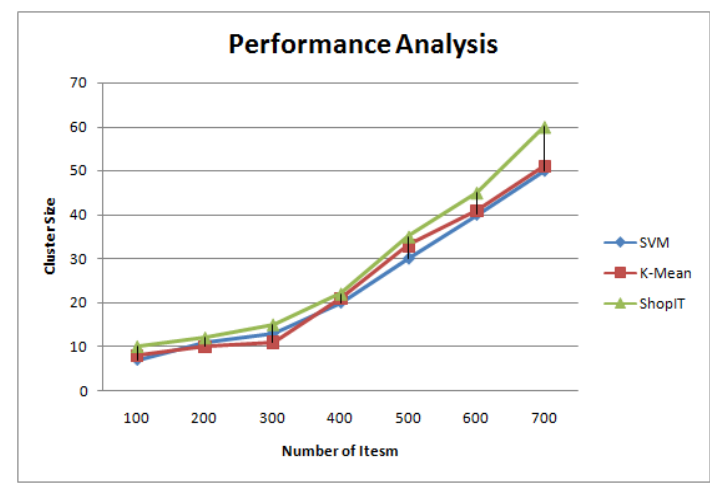

Figure 2 Efficiency of the proposed method.

\section{CONCLUSION}

E-commerce is crucial tool in a human life. But grouping and scheduling for shipment creates a lot of challenges. The time delay in order processing and order fulfillment is challenging issue. In this a tool "Shop IT" is introduced to address the above problem. It is two phase model in phase one greedy algorithm is used to A Route Mapper is used to find the shortest path. In phase-II optimum grouping algorithm is used to form the clusters. The experimental results show the efficiency of the system.

\section{REFERENCES}

1. Abdul Gaffar Khan," Electronic Commerce: A Study on Benefits and Challenges in an Emerging E-economy", global Journal of Management and Business Research, Vol. 6, Issue. 1, 2016.

2. Bhupinder Singh and Ashwini Seth, AN Analysis of Security using ECDH in E-Commerce, - International Journal for Scientific Research \& Development, Vol. 4, Issue 9, 2016.

3. Nisha Chanana and Sangeeta Goele, Future of E-Commerce in India, International Journal of Computing \& Business Research, 2002.

4. D. K. Gangeshwer, E-Commerce or Internet Marketing: A Business Review from Indian Context, International Journal of u- and e- Service, Science and Technology Vol.6, No.6, pp.187-194, 2003. 
5. M.Shirisha, Digital Marketing Importance in the New Era, International Journal of Engineering Technology Science and Research, Vol. 5, Issue 1, 2018.

6. Diyan Ivanov, Lokesh Giripunje and Sonali Nimbhorkar, Comprehensive Security System for Mobile Network Using Elliptic Curve Cryptography over GF (p), International Journal of Advanced Research in Computer Science and Software Engineering Vol. 3, Issue 5, 2013.

7. Lokesh Giripunje and Sonali Nimbhorkar, Comprehensive Security System for Mobile Network Using Elliptic Curve Cryptography over GF (p), International Journal of Advanced Research in Computer Science and Software Engineering Vol. 3, Issue 5, 2013.

8. N. Jayakanthan, M. Manikantan and R. Vivek, the enhanced learning model to improve the quality of E-Shopping and stock management ", International Journal of Pure and Applied Mathematics, Vol. 115, No. 2, 2018, pp. 837-841.

9. N. Jayakanthan and A. V. Ramani, Graph based Classifier to Detect Malicious URLs, International Journal of Mechanical and Production Engineering Research and Development Vol.7, Issue.5, pp. 223-234, 2017 